\title{
Surface polish of PLA parts in FDM using dichloromethane vapour
}

\author{
Yifan Jin, Yi Wan and Zhanqiang Liu \\ Key Laboratory of High Efficiency and Clean Manufacturing, School of Mechanical Engineering, Shandong University, Jinan, China
}

\begin{abstract}
Fused deposition modelling has become one of the most diffused rapid prototyping techniques, which is widely used to fabricate prototypes. However, further application of this technology is severely limited by poor surface roughness. Thus it is necessary to adopt some operations to improve surface quality. Chemical finishing is typically employed to finish parts in fused deposition modelling (FDM). The purpose of this paper is to decrease the surface roughness for polylactic acid (PLA) parts in FDM. The chemical reaction mechanism during the treating process is analysed. Then $\mathrm{NaOH}$ solution and dichloromethane vapour are used to treat FDM specimens respectively. A 3D laser microscope has been applied to assess the effects in terms of surface topography and roughness. The experimental results show that treatment using dichloromethane vapour performs much better than $\mathrm{NaOH}$ solution. Compared with the untreated group, surface roughness obtained through vapour treatment decreases by 88 per cent. This research has been conducted to provide a better method to treat PLA parts using chemical reagents.
\end{abstract}

\section{Introduction}

Fused deposition modelling (FDM) is the most diffused rapid prototyping (RP) technique in the world ${ }^{[1]}$. The main advantages of this technology include: low maintenance cost, toxic free materials, quick production of complexity parts, and high utilization rate of raw materials. These aspects make it suitable for a wide range of applications in aerospace, automotive, biomedical, customer product industry, design and tooling [2-4]. Nevertheless, the main disadvantages are anisotropic property, requirement of support structures for overhangs and high surface roughness ${ }^{[5]}$. Particularly, the poor surface limits the further application.

Several researches have been investigated to reduce surface roughness of FDM parts by optimizing fabrication parameters. A study conducted by Thrimurthulu ${ }^{[6]}$ et al. aimed to obtain an optimum part deposition orientation by using a real coded genetic algorithm. Ahn ${ }^{[7,8]}$ et al. proposed a model to represent average roughness by assuming the filament profile as an elliptical curve, and regarded cross-sectional shape, surface angle, layer thickness and overlap between adjacent layers as the main factors affecting surface quality. An approach for adaptive slicing that applied the image processing technique to determine appropriate thickness and to recommend slicing positions on a 3D model was proposed, then it was implemented on the LabVIEW platform and compared with other approaches to reduce build time while maintaining surface quality ${ }^{[9]}$. Mahapatra and Sood ${ }^{[10]}$ proposed use of artificial neural network model to determine the relationship between five input parameters, subsequence bayesian regularization was adopted for selection of optimum network architecture to obtain reasonably good surface quality.

Whereas, roughness on the vertical orientation caused by staircase effect cannot be avoided through optimizing fabrication parameters. Hence, post-treatment operations to improve surface quality is needed.

At present, two primary approaches are used to achieve smooth surfaces on parts: chemical and mechanical smoothing ${ }^{[11]}$. A chemical post-treatment was adopted after studying the influence of FDM machining parameters on acrylonitrile butadiene styrene (ABS) prototypes surface finish, subsequently, surface finish of products had been measured, which showed a significant improvement in surface finish at the expense of a negligible change in the dimensional accuracy ${ }^{[12]}$. Application of surface coatings is another approach to achieve a desired surface finish, in addition to adding strength to a finished part. Such coatings may include primers, paints or metallic electroplated coatings ${ }^{[13]}$.

However, chemical polish of polylactic acid (PLA) has been rarely investigated, not to mention using different chemical reagents.

In this paper, two distinct chemical reagents are adopted to reduce surface roughness of PLA parts respectively, with one method using $\mathrm{NaOH}$ solution, and the other using dichloromethane $\left(\mathrm{CH}_{2} \mathrm{CL}_{2}\right)$ vapour. Subsequently, the surface topography and roughness are observed by a 3D laser scanning microscope to verify the mechanism of chemical reaction analysed.

\section{Theory analysis}




\subsection{Effects of fabrication parameters}

Many fabrication parameters have impacts on the surface quality of FDM parts, such as layer thickness, nozzle diameter, printing speed, raster angle, shell thickness and infill overlap etc., which are shown in Figure 1. Moreover, different factors make diverse influences on the surface quality. The major ones are layer thickness and nozzle diameter. The thin layer produces a smoother surface than the thick layer, whether it is measured vertically or horizontally ${ }^{[14]}$. Surface roughness and part deposition time are always contradicted with each other. It is the same with nozzle diameter. However, in any case the staircase effect on the vertical orientation cannot be eliminated by optimizing fabrication parameters.

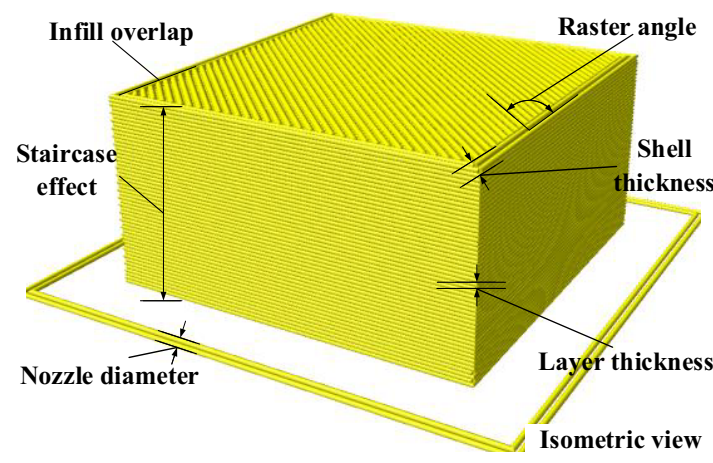

Figure 1. Illustration of partial fabrication factors affecting surface quality

\subsection{Effects of fabrication parameters}

Opposite to the polycondensation of esters, there are three methods during the hydrolysis process of PLA: (i) occurring on the main chain to generate monomers or oligomers, (ii) hydrolysis on the side chain to generate soluble main chain polymer and (iii) hydrolysis on the linkage chain to generate linear polymer ${ }^{[15]}$. And the principal way is (i), as shown in Figure 2. The hydrolysis of PLA begins with the absorption of water, then small molecules of water move to the surface of the sample, spreading around the ester bond or hydrophilic group. Especially in the medium of acid or alkali, the ester bond in macromolecular main chain is broken continually. Ultimately samples dissolve when the molecule reduces to a certain extent. Furthermore, the characteristic of this process is that the hydrolysis happens at the interface simultaneously, and small molecules generated leave the model surface to diffuse in aqueous solution.

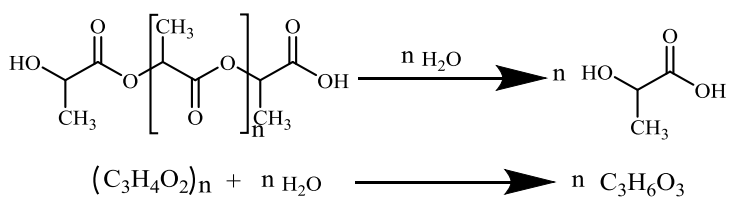

Figure 2. Illustration of hydrolysis of PLA

\subsection{Dissolution of PLA in organic solvent}

For FDM parts made up of PLA, there exists a range of corrugated structures on the surface due to the staircase effect, which is supposed to be eliminated by using vapour treatment of easy volatile solvent such as chloroform, dichloromethane and tetrahydrofuran etc. Ahn ${ }^{[16]}$ et al. has proposed a magnified cross-sectional shape of the deposited filaments, which is close to an elliptic curve. Appear on model surface, peaks and valleys become droplets through absorbing the volatile solvent. Then, under the effects of liquid surface tension and gravity, these two kinds of droplets blend continually, resulting in lower peak and filled valley. As it goes on, a dynamic balance will be achieved. Finally, a smoother surface can be obtained. The process of dissolving and flow on the profile of PLA part is shown in Figure 3. Laser Raman spectra shows that there exists no dichloromethane in the dried product, and it has been volatilized completely during this processing ${ }^{[17]}$. The main distinction compared with hydrolysis of PLA is that the dissolved molecules attach to the model surface during the progress.

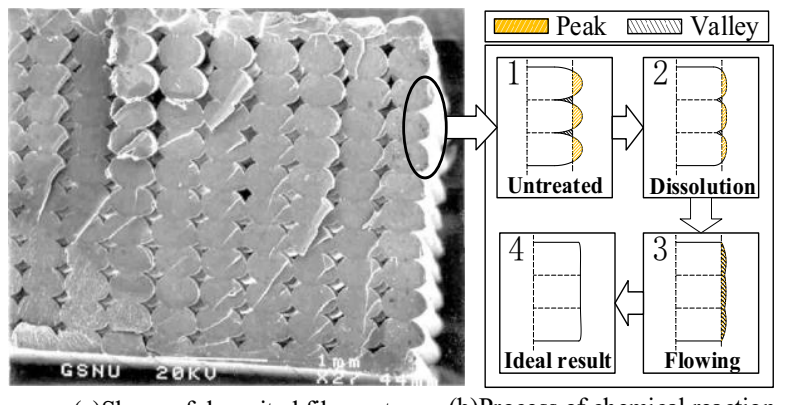

(a)Shape of deposited filaments (b)Process of chemical reaction Figure 3. Process of dissolution and flow on the profile of PLA part. (a) Shape of deposited filaments ${ }^{[16]}$ and (b) process of chemical reaction.

\section{Experimental}

In this paper, two chemical methods for enhancing the surface finish of specimens are investigated. The $\mathrm{NaOH}$ solution is adopted in order to treat the surface of specimens in the first method, and dichloromethane is used as the organic solvent in the second method. The progress is carried out over three steps. Figure 4 displays the workflow of the experiment.

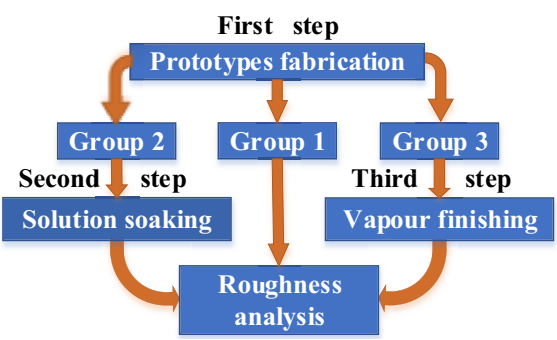

Figure 4. Experimental workflow

\subsection{First step -specimens manufacture}

A cuboid test specimen is designed and its dimension is shown in Figure 5 with the unit used is millimetre. All specimens for groups of three are fabricated using a Prusa 
Mendel desktop 3D printer, and the filament feedstock is PLA. Printing parameters are adopted by empirical values in order to eliminate the manufacture error. Table 1 shows the main printing factors above-mentioned in Chapter 2.1. Other factors are maintained at their default levels.

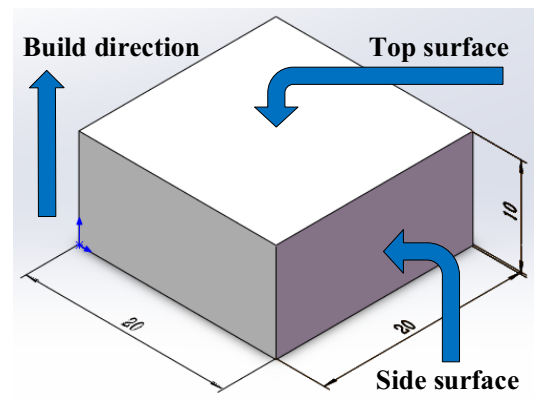

Figure 5. Shape and dimension of specimens

Table 1. Printing factors and their levels

\begin{tabular}{|c|c|c|}
\hline \multicolumn{1}{|c|}{ Factor } & Level & Unit \\
\hline Layer thickness & 0.2 & $\mathbf{~ m m}$ \\
\hline Raster angle & 45 & $\circ$ \\
\hline Nozzle diameter & 1.0 & $\mathbf{~ m m}$ \\
\hline Printing speed & 30 & $\mathbf{~ m m} / \mathbf{s}$ \\
\hline Shell thickness & 1.0 & $\mathbf{m m}$ \\
\hline Infill overlap & $10 \%$ & - \\
\hline
\end{tabular}

\subsection{Second step - treatment with $\mathrm{NaOH}$ solution}

In the second step of the experimentation, the specimens of group two were subjected to treatment of soaking in $\mathrm{NaOH}$ solution. Heating by an alcohol lamp is added for the reason that reaction performs quite slow at room temperature. The process was carried out by immersing in the 5 wt. $\% \mathrm{NaOH}$ boiling solution for half an hour. Subsequently, the roughness of side and top surfaces of all the specimens were measured by the laser microscope for 3D \& profile measurement, in order to evaluate any differences happened. Three specimens were assessed to avoid the accidental error.

\subsection{Third step - treatment with dichloromethane vapour}

In the third step, the specimens of group three were subjected to an organic chemical treatment. The dichloromethane was selected according to its low cost, very low toxicity and extremely high diffusion. Before the test, a processing time trial was made to decide the suitable treatment time. The process was undertaken in a glass bottle full of dichloromethane vapour for $300 \mathrm{~s}$. The operation was conducted in room temperature at $25^{\circ} \mathrm{C}$ and the pressure of vapour using is at normal atmospheric pressure. The surface roughness of three specimens was evaluated using the same laser microscope and compared with the above two groups in order to evaluate the improvement of surface finish.

\section{Results and discussion}

\subsection{Analysis of surface roughness}

Surface roughness were measured by a laser scanning microscope (KEYENCE VK-X200). Images were taken over a $1.414^{*} 1 \mathrm{~mm}$ area of the side surface. Surface roughness was analysed by the VK Analyzer 3.3 software (KEYENCE, JAPAN).

In Figure 6, the 3D surface topography and its line roughness of side surfaces are shown. Three colourful lines show the position of the line roughness, while the corresponding curves below represent the line roughness measured. Surface defects near the edges were avoided.

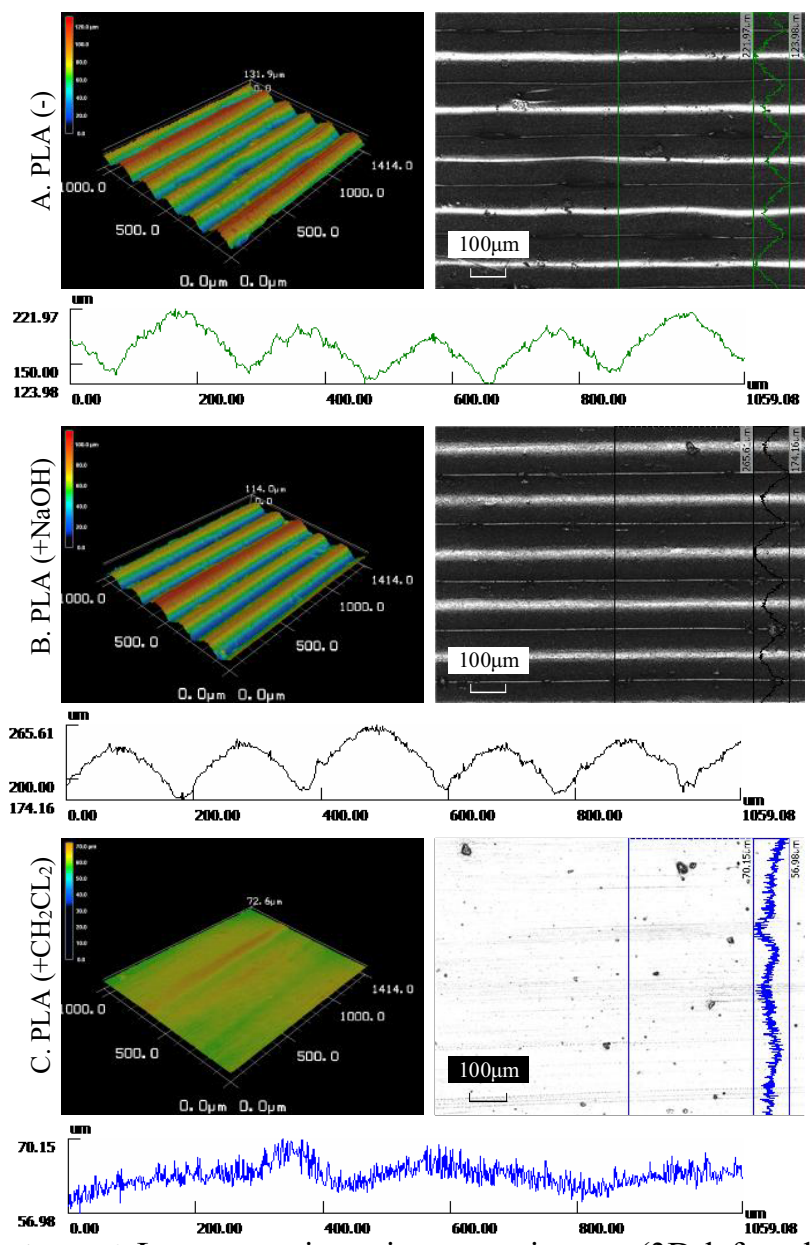

Figure 6. Laser scanning microscope images (3D-left and 2D-right) of the side surface showing before (-) and after $(+)$ chemical treatments. (A) Untreated PLA, (B) treated PLA $(+\mathrm{NaOH}),(\mathrm{C})$ treated PLA $\left(+\mathrm{CH}_{2} \mathrm{CL}_{2}\right)$. All images are at the same scale.

As shown in Figure 6, the roughness of the specimens in group three have been greatly reduced by using the dichloromethane vapour treatment described 
above. Roughness before and after chemical treatments is reported in Table 2. Compared with group one, the surface roughness of group two reduced by 13 per cent. However, the surface treated by $\mathrm{NaOH}$ solution loses its lustre which can be obviously seen in the middle of Figure 6. Further investigation on the nano-level needs to be conducted. An upgrade reflection effect of light is obtained in group three. Meanwhile, the surface line between neighbour layers cannot be observed by the naked eye. A markedly roughness reduction is obtained with a lowering of 88 per cent than group one, and 86 per cent than group two, which indicates the treatment used in group three performs much better than that used in group two. In other words, the method using dichloromethane vapour almost eliminates the influence of staircase effect. As to the top surface, the results show that there is poorly difference amongst the three groups. The roughness of top surface is decided by many factors except the staircase effect. Hence, the measured results have been elided in this paper.

Table 2. Roughness on side surface of three groups

\begin{tabular}{|c|c|c|c|c|}
\hline & $\begin{array}{c}\text { Group } \\
\text { number }\end{array}$ & $\begin{array}{c}\text { Specimens } \\
\text { number }\end{array}$ & $\begin{array}{c}\text { Ra } \\
(\mu \mathrm{m})\end{array}$ & $\begin{array}{l}\text { Average } \\
\operatorname{Ra}(\boldsymbol{\mu} \mathbf{m})\end{array}$ \\
\hline \multirow{3}{*}{$\begin{array}{c}\text { Before } \\
\text { treatment }\end{array}$} & \multirow{3}{*}{ I } & 1 & 18.84 & \multirow{3}{*}{20.46} \\
\hline & & 2 & 22.33 & \\
\hline & & 3 & 20.20 & \\
\hline \multirow{3}{*}{$\begin{array}{c}\text { After } \\
\text { NaOH } \\
\text { treatment }\end{array}$} & \multirow{3}{*}{ II } & 4 & 17.34 & \multirow{3}{*}{17.79} \\
\hline & & 5 & 19.15 & \\
\hline & & 6 & 16.92 & \\
\hline \multirow{3}{*}{$\begin{array}{c}\underset{\text { After }}{\mathrm{CH}_{2} \mathrm{CL}_{2}} \\
\text { treatment }\end{array}$} & \multirow{3}{*}{ III } & 7 & 1.90 & \multirow{3}{*}{2.46} \\
\hline & & 8 & 2.47 & \\
\hline & & 9 & 3.00 & \\
\hline
\end{tabular}

In Boschetto and Bottini ${ }^{[18]}$, through barrel finishing, the $\mathrm{Ra}$ decreases from 16.54 to $4.23 \mu \mathrm{m}$ in 960 minutes for vertical walls of ABS FDM parts. In this paper, the results show that after vapour smoothing for just 30 seconds the peak and valley of the surface cannot be distinguished any more, with average $\mathrm{Ra}$ passes from 20.46 to $2.46 \mu \mathrm{m}$. However, there still exists a tiny uneven area as shown at the bottom of Figure 6. The reason for this phenomenon is unknown. Better surfaces may be produced if a regularly vibrating treatment applied on the specimens is adopted simultaneously with vapour treatment, which may promote the flowing droplets to homogenize.

\subsection{Case study}

The investigation of this paper has proposed a chemical method to improve the surface finish, which can be used to fabricate parts. To provide a case of treatment on free form surfaces, a cartoon character (Figure 7 - left side) was manufactured using the vapour post-treatment. Then the prototype was immersed in the chemical vapour for thirty seconds and its roughness was measured on a $1.414 * 1 \mathrm{~mm}$ area considered on the front part. The roughness reduces from an average $\mathrm{Ra}$ equal to $18.24 \mu \mathrm{m}$ to $3.77 \mu \mathrm{m}$. The $3 \mathrm{D}$ map and 2D surface topography are shown in Figure 7 - right side. It can be observed that the surface quality has been greatly improved.

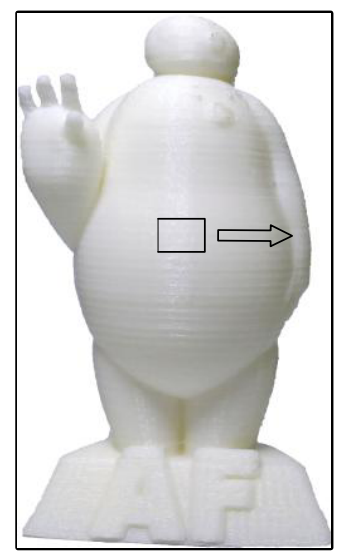

(a)

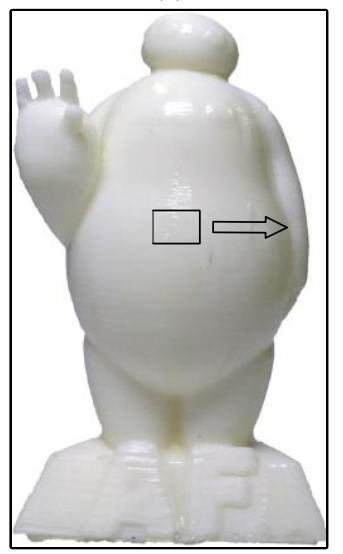

(b)

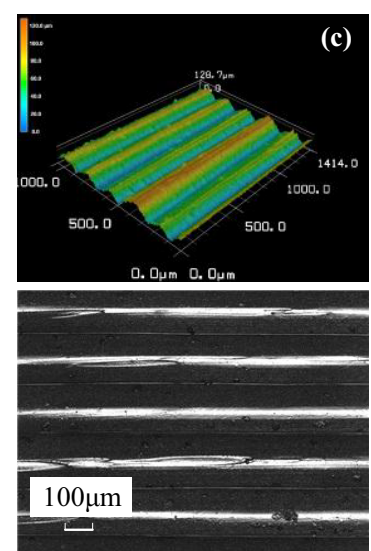

(d)

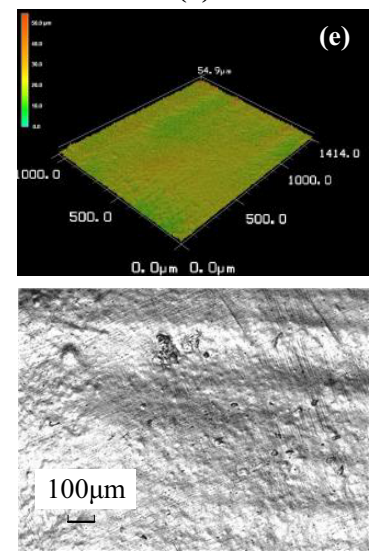

(f)
Figure 7. Images of free form surface for (a) untreated and (b) treated by dichloromethane vapour

\section{Conclusion}

In this paper, the chemical reaction process of FDM prototypes made up of PLA is analysed originally. Using different chemical reagents, two methods of improving the surface finish are investigated, with vapour treatment yielding a significant enhancement of surface roughness. Compared with untreated specimens, average $\mathrm{Ra}$ is found to decrease by 88 per cent using dichloromethane vapour and near mirror quality surfaces can be obtained regardless of staircase effect. While the specimens treated by $\mathrm{NaOH}$ solution can also decrease average $\mathrm{Ra}$ in some degree. The proposed chemical treatment is economic, fast and easy to use. With simple treatment and little time, the experimental findings provide the operator a method to polish PLA parts, which can reduce instrument and labour cost in the post-processing operation. Further 
studies need to be conducted on the mechanical property after vapour treatment, particularly on the vertical orientation.

\section{Acknowledgements}

The authors would like to acknowledge the financial support from National Natural Science Foundation of China (51175306 and 51575320), Tai Shan Scholar Foundation and Fundamental Research Funds for the Central Universities (2014JC020).

\section{References}

1. Wohlers, T. (2012), "Executive Summary", Wohlers Report, Wohlers Associates, Fort Collins, CO. Wohlers, T. (2012), "Executive Summary", Wohlers Report, Wohlers Associates, Fort Collins, CO.

2. Chua, C.K., Leong, K.F. and Lim, C.S. (2010), Rapid Prototyping: Principles and Applications, World Scientific, River Edge, NJ.

3. Ingole, D.S., Kuthe, A.M., Thakare, S.B. and Talankar, A.S. (2009), "Rapid prototyping - a technology transfer approach for development of rapid tooling", Rapid Prototyping Journal, Vol. 15 No. 4, pp. 280-290.

4. Ivanova, O., Williams, C. and Campbell, T. (2013), "Additive manufacturing (AM) and nanotechnology: promises and challenges", Rapid Prototyping Journal, Vol. 19 No. 5, pp. 353-364.

5. Brooks, H., Lupeanu, M.E. and Piorkowski, B. (2013), "Research towards high speed extrusion freeforming", International Journal of Rapid Manufacturing, Vol. 3 Nos 2/3, pp. 154-171.

6. Thrimurthulu, K., Pandey, P.M. and Reddy, N.V. (2004), "Optimum part deposition orientation in fused deposition modeling", International Journal of Machine Tools and Manufacture, Vol. 44 No. 6, pp. 585-594.

7. Ahn, D.K., Kim, H. and Lee, S. (2009a), "Surface roughness prediction using measured data and interpolation in layered manufacturing", Journal of Materials Processing Technology, Vol. 209 No. 2, pp. 664-671.

8. Ahn, D.K., Kweon, J.H., Kwon, S., Song, J. and Lee, S. (2009b), "Representation of surface roughness in fused deposition modelling", Journal of Materials
Processing Technology, Vol. 209 Nos 15/16, pp. 5593-5600.

9. Rianmora, S. and Koomsap, P. (2010), "Recommended slicing positions for adaptive direct slicing by image processing technique", The International Journal of Advanced Manufacturing Technology, Vol. 46 Nos 9/10/11/12, pp. 1021-1033.

10. Mahapatra, S.S. and Sood, A.K. (2012), "Bayesian regularization-based Levenberg-Marquardt neural model combined with BFOA for improving surface finish of FDM processed part", The International Journal of Advanced Manufacturing Technology, Vol. 60 Nos 9/10/11/12, pp. 1223-1235.

11. Gibson, I., Rosen, D.W. and Stucker, B. (2010), Additive manufacturing technologies: rapid prototyping to direct digital manufacturing, Springer, New York, NY.

12. Galantucci, L., Lavecchia, F. and Percoco, G. (2009), "Experimental study aiming to enhance the surface finish of fused deposition modeled parts", CIRP Annals-Manufacturing Technology, Vol. 58 No. 1, pp. 189-192.

13. Turner, B.N., Strong, R. and Gold, S.A. (2014), "A review of melt extrusion additive manufacturing processes: I. Process design and modeling", Rapid Prototyping Journal, Vol. 20 No. 3, pp. 192-204.

14. Bakar, N.S.A., Alkahari, M.R. and Boejang, H. (2010), "Analysis on fused deposition modelling performance", Journal of Zhejiang University Science A, Vol. 11 No. 12, pp. 972-977.

15. Siparsky, G.L., Voorhees, K.J. and Miao, F. (1998), "Hydrolysis of polylactic acid (PLA) and polycaprolactone (PCL) in aqueous acetonitrile solutions: autocatalysis", Journal of environmental polymer degradation, Vol. 6 No. 1, pp. 31-41.

16. Ahn, S.-H., Montero, M., Odell, D., Roundy, S. and Wright, P.K. (2002), "Anisotropic material properties of fused deposition modeling ABS", Rapid Prototyping Journal, Vol. 8 No. 4, pp. 248-257.

17. Kasuga, T., Fujikawa, H. and Abe, Y. (1999), "Preparation of polylactic acid composites containing $\beta-\mathrm{Ca}$ (PO3)2 fibers", Journal of materials research, Vol. 14 No. 2, pp. 418-424.

18. Boschetto, A. and Bottini, L. (2015), "Surface improvement of fused deposition modeling parts by barrel finishing", Rapid Prototyping Journal, Vol. 21 No. 6, pp. 686-696. 